\title{
Pluralismo constitucional y espacios transnacionales: ¿el fin de la constitución nacional o su nuevo comienzo? ? $^{* * *}$
}

\section{Constitutional pluralism and trasnational spaces: The end or a new beginning of the National Constitution?}

RESUMEN

El modelo clásico de organización política atraviesa un proceso de crisis profunda. Inexorablemente, las “fronteras" políticas, económicas, jurídicas, sociales y culturales de los Estados se tornan cada vez más porosas (para no decir sin sentido). Esta situación hace que las diferenciaciones entre derecho y política modernamente operadas por la constitución se diluyan, hasta el punto de que los elementos conceptuales clásicamente formulados por la teoría constitucional se muestran ahora incapaces de resolver los problemas relativos a la normativa social y las pretensiones de ordenación social. Este artículo pretende discutir en qué medida es posible mantener las funciones tradicionales asignadas a la constitución en vista de la ampliación de las áreas de regulación transnacional. Trata, en otras palabras, de entender las consecuencias y las posibilidades de (re)organización del proceso de ordenación que afectan al diseño estructural de la soberanía constitucional en su forma más fundamental, es decir, en su capacidad soberana para decidir en un determinado territorio. Se hace énfasis en la dimensión institucional de estas

Doctora en Derecho por la Universidad Federal de Santa Catarina (UFSC) (Brasil). Profesora Asociada de Teoría y Filosofia de Derecho de la Universidad Federal do Rio de Janeiro, se desempeña en el área de Teorías Jurídicas Contemporáneas (línea de investigación: Diseños institucionales). Orientadora de los programas de maestría y doctorado. Becaria de productividad en investigación científica (CNPq/PQ2). Coordinadora de proyectos de investigación y extensión en derecho constitucional y filosofía del derecho y de la política. Contacto: cecilialois@ gmail.com

Pos-doctor en Derechos Humanos por la Universida McGill. Doctor en Derecho por la Universidad Federal de Santa Catarina (UFSC) (Brasil). Profesor de Derecho Constitucional y Derechos Humanos de los cursos de Derecho y Relaciones Internacionales de la Universidad de Vale do Itajaí (univali). Profesor del Programa de Maestría y Doctorado en Ciencia Jurídica de la UNIVALI. Contacto: lmagno@univali.br; luiz.magno.jr@gmail.com

**** Recibido el 26 de julio de 2017, aprobado el 15 de octubre de 2017.

Para citar el artículo: CABAllero Lois, C. y Pinto Bastos Júnior, L. M. Pluralismo constitucional y espacios transnacionales: ¿el fin de la constitución nacional o su nuevo comienzo? Derecho del Estado n. ${ }^{\circ}$ 40, Universidad Externado de Colombia, enero-junio de 2018, pp. 127151. DOI: https://doi.org/10.18601/01229893.n40.06 
transformaciones, con vistas a formular, al final de este artículo, las bases para la identificación de un modelo de pluralismo constitucional apoyado sobre las máximas de pluralidad, ordenación heterárquica y conflictividad.

\section{PALABRAS CLAVE}

Espacios transnacionales, pluralismo constitucional, transición de modelos de ordenación constitucional.

\section{ABSTRACT}

This article aims to discuss to what extent it is possible to maintain the traditional function assigned to the constitution when it is confronted with the expanding the areas of transnational regulation. In other words, it to understand the consequences and possibilities of (re)organization of the ordering process that affects the structural design of constitutional sovereignty in its most fundamental feature, that is, its sovereign capacity to decide in a given territory.

\section{KEYWORDS}

Transnational spaces, constitutional pluralism, transition on models of constitutional ordering.

SUMARIO

Introducción. 1. Los desafíos del pluralismo jurídico al constitucionalismo contemporáneo. 2. Del principio de jerarquización normativa a la idea-fuerza de heterarquía. 3. De la coherencia intrasistemática a la exigencia de compatibilización: en busca de nuevos parámetros para la supraordenación en estructuras heterárquicas. A modo de conclusión: pluralismo constitucional como respuesta posible a las presiones sobre la constitución nacional. Referencias.

\section{INTRODUCCIÓN}

El presente artículo tiene por finalidad discutir en qué medida es posible preservar las funciones tradicionales atribuidas a la constitución en el proceso de configuración de espacios de regulación transnacional ${ }^{1}$. Se trata, en otros

1 Los "espacios" de regulación transnacional ocupan una posición intermedia entre lo nacional y lo internacional (in-between state). Esta condición intermediaria se presenta justamente porque estos espacios normativos se forman mediante procesos de negación y de diferenciación con relación a los ámbitos normativos típicamente estatales; se refieren a espacios "construidos" por redes de inter-relaciones que se proyectan más allá del Estado. La idea de un "espacio 
términos, de comprender cuáles son las consecuencias y las posibilidades de (re)organización del constitucionalismo resultantes de los procesos de globalización y de creciente interdependencia que alcanzan la concepción estructurante de soberanía constitucional en su elemento más central, a saber, su capacidad de decisión soberana en determinado territorio, indicada por la constitución.

En efecto, la comprensión de la constitución como criterio para la demarcación de las fronteras de validez del orden jurídico estatal fue construida con base en tres atributos que le son usualmente conferidos en su condición de "estatuto orgánico de lo político": la territorialidad como criterio de demarcación espacial de validez; la unidad sistemática del ordenamiento jurídico; y, la legitimidad para fijar la unión jerárquica de las autoridades. Estos elementos son centrales en la noción de Estado territorial soberano y pueden ser perfectamente traducidos por las ideas conexas de "monismo jurídico-estatal" y "estructura piramidal del ordenamiento jurídico".

A lo largo del proceso de formación y de consolidación de este modelo de organización centrado en el Estado, el acoplamiento entre las categorías de territorio, soberanía y constitución contribuyó para que la relación entre el derecho y la política sufriese una triple diferenciación/reducción: (i) una diferenciación entre lo público y lo privado y la reducción de la dimensión pública al elemento estrictamente estatal; (ii) una diferenciación entre el derecho y el no derecho y la reducción de aquellos a las formas jurídicas producidas en consonancia con las instancias oficiales; y, (iii) una diferenciación entre los espacios interno y externo, demarcada por la creación artificial de las fronteras y la reducción de aquel que sería normativamente relevante a los elementos producidos en el interior de los Estados territoriales.

Sin embargo, este modelo de organización política atraviesa un proceso de crisis profunda. Inexorablemente, las "fronteras" políticas, económicas, jurídicas, sociales y culturales de los Estados se tornan cada vez más porosas (para no decir sin sentido). Esta situación hace que las diferenciaciones entre derecho y política modernamente operadas por la constitución se diluyan hasta tal punto que los elementos conceptuales clásicamente formulados por la teoría constitucional se muestran incapaces de resolver los problemas referidos a la normativa social y a las pretensiones de ordenación social.

Este escenario exige que se discuta la posibilidad de mantener operativa la fórmula soberana (summa potestas) delante de algunos de estos fenómenos, sobre todo cuando se pretende justificar normativamente un "deber de

transnacional" debe ser comprendida como una zona de interacción que no se construye sobre bases necesariamente territoriales. Estos espacios, concebidos en términos funcionales y siempre relacionales, emergen en el exacto momento en que las relaciones entre los diferentes dominios normativos cobran cuerpo. El espacio transnacional se constituye, pues, mediante interacciones, surge -siempre- en la interacción (BASTOS Jr., 2011, 168-182). 
apertura" de los sistemas jurídicos nacionales a elementos que les son, en principio, externos. El gran desafío contemporáneo al constitucionalismo consiste justamente en lograr definir parámetros para la regulación de los conflictos a partir de reglas de colisión que no se asienten, en últimas, en la autoridad territorialmente jerarquizada, sino en la capacidad de la decisión de producir puntos de convergencia material.

En este trabajo se defiende abiertamente que las funciones modernamente atribuidas a la constitución ${ }^{2}$ (como factor de integridad ${ }^{3}$ y de integración ${ }^{4}$ de la comunidad política) permanecen operativas siempre y cuando los múltiples discursos sobre la constitución sean formulados en el marco de la preocupación por la preservación de las diferencias. Este compromiso se traduce, a su vez, en la formación de múltiples redes de interacción y en la necesidad de ampliación de los mecanismos de diálogo institucional con diferentes actores (sobre todo, aquellos ubicados fuera del Estado).

Al señalar esta dirección se pretende mostrar que se está ante una situación paradójica en la cual la única forma de mantener la autoridad del Estado y su más eficaz elemento de justificación (la idea de soberanía) consiste en el reconocimiento de límites inmanentes y de mecanismos de compartimiento de autoridad soberana.

Trasladando esta cuestión al constitucionalismo, esto quiere decir que la única forma que se vislumbra para que el potencial de garantía y ordenador de los discursos sobre la constitución sea mantenido consiste en desconcentrar la autoridad constitucional y reconocer la existencia de centros diversificados de producción de discursos constitucionales. Estos centros de decisiones diferenciados se ubican más allá de las fronteras jurídicas de la constitución

2 A fin de esclarecer la perspectiva adoptada, es preciso señalar que la concepción de constitución es asumida en este trabajo como un conjunto multifacético de discursos institucionalizados revestidos de capacidad de ordenación social en "una" determinada comunidad política. Por consiguiente, la constitución no se reduce solamente al texto constitucional, sino que lo extrapola. Como texto normativo, su sentido depende siempre de procesos concretos de atualización que re-insertan el contexto en el interior del programa normativo. Estos procesos de actualización, a su vez, son igualmente enunciados a través de nuevas fórmulas linguísticas, hecho este que desencadena nuevos ciclos de actualización textual a cada aplicación concreta. Así, "una" constitución puede ser descrita como obra siempre inacabada, con lagunas, contradictoria y permeada por desacuerdos y conflictos ineliminables.

3 La constitución como factor de integridad representa el reconocimiento de su función nomogenética y de su aptitud para definir el fundamento de validez normativa del ordenamiento jurídico (dimensión organizativa de la constitución). De esta forma, el atributo de validez de cualquier disposición normativa debe guardar relación de compatibilidad vertical con las reglas fundantes definidas en el documento constitucional. El derecho válido se estructura, a partir del Estado, con base en las ideas reguladoras de jerarquía, unidad y coherencia.

4 La constitución como factor de integración representa el reconocimiento de que esta cumple un relevante papel como factor de ordenación social (dimensión valorativa de la constitución). Por consiguiente, la tensión continua y constante entre normatividad y normalidad debe ser incorporada a la comprensión del sentido y del alcance del documento constitucional. 
nacional, superan las barreras estatales y se afirman discursivamente en el plano doméstico de los Estados.

Este escenario suscita dos problematizaciones relacionadas y coimplicadas: una de carácter sustantivo (¿cómo conferir legitimidad a las decisiones?) y otra de naturaleza eminentemente institucional (¿cómo hacer frente a la existencia de autoridades en competencia?). La cuestión central sobre la "capacidad de decidir" no puede construirse más con base en el elemento constitutivo de la territorialidad, sino en la capacidad de la norma/decisión de producir puntos de convergencia material.

Aquí se pretende hacer énfasis en la dimensión institucional de estas transformaciones, o sea, en los siguientes procesos de transición: (i) de la noción estructurante de unidad sistémica (a partir del derecho estatal) a la necesidad de reconocimiento de distintas fuentes normativas igualmente legítimas (producidas más allá del Estado); (ii) de la pretensión de ordenación jerárquica a la necesidad de acomodación de diferentes regímenes jurídicos multinivel y segmentados; (iii) de la consagración del dogma de la coherencia intrasistémica (deber de corrección y eliminación de las antinomias) al reconocimiento de las diferencias y de la pluralidad como valor que exige esfuerzos de compatibilización de normas en conflicto, sin resultar en la eliminación necesaria de estas antinomias. Elementos de transición estos que serán enfrentados en las próximas secciones de este artículo.

La identificación de estos esfuerzos de cambio, según se pretende demostrar en el final de este artículo, permite formular las bases para la identificación de un modelo de pluralismo constitucional apoyado sobre las máximas de pluralidad, ordenación heterárquica y conflictividad.

\section{LOS DESAFÍOS DEL PLURALISMO JURÍDICO AL CONSTITUCIONALISMO CONTEMPORÁNEO}

En esta primera sección se pretende señalar de qué forma la idea de unitas multiplex puede ser capaz de mantener la pretensión regulatoria de la constitución nacional, cuando esta misma constitución reconoce la existencia simultánea de múltiples regímenes jurídicos, en un mismo espacio normativo $^{5}$. Con el fin de ofrecer algunos subsidios preliminares para enfrentar esta cuestión específica se harán, inicialmente, breves incursiones en los debates contemporáneos en torno del pluralismo jurídico y los desafíos que este plantea a la teoría del derecho.

5 Esta cuestión puede ser problematizada en los siguientes términos: ¿cómo puede la constitución (nacional) ser capaz de operar como factor de integridad y de integración si admite la existencia legítima, en un mismo dominio territorial, de regímenes jurídicos con fundamentos de validez distintos? 
El pluralismo jurídico puede ser genéricamente definido como una "concepción que se refiere a órdenes, redes y sistemas jurídicos coexistentes en un mismo espacio geográfico" (Avbeli, 2006, 378). Esta expresión encierra contemporáneamente múltiples usos y viene ganando proyección en diversos dominios científicos (antropología jurídica, derecho comparado, derecho internacional, estudios sobre globalización) (Tamanaha, 2008, 3).

$\mathrm{Si}$, por un lado, esta profusión de usos refuerza la narrativa en torno de la necesidad de redimensionar la forma de comprender la relación entre el derecho oficial (estatal) y los demás patrones de regulación social embebidos en distintos fragmentos de las comunidades (subnacionales y transnacionales), por otro lado pone en evidencia innumerables dificultades conceptuales y el desafío constante de producir puentes de comunicación entre los diferentes dominios científicos de las ciencias sociales (Dupret, 2007).

De acuerdo con Brian Tamanaha (2008, 25-26), la actual agudización del debate en torno del pluralismo jurídico (a escala global) deriva de dos cambios recientes de perspectiva: (i) de la fijación de los niveles global y transnacional de regulación como puntos de partida de los análisis, hecho este que coloca a las divergencias y conflictos suscitados como aspectos centrales de los fenómenos que han de ser comprendidos; y, (ii) de un cambio realizado por los juristas, en el sentido de incorporar una visión más amplia del derecho (como fenómeno social ampliado), el cual acaba por "producir" una "profusión de órdenes jurídicos".

Es posible identificar un conjunto muy diferenciado de propuestas teóricas en torno del pluralismo jurídico que, de acuerdo con los propósitos de este trabajo, pueden ser agrupadas a partir de tres perspectivas distintas: una primera, que reúne los estudios que se ocupan, de forma muy amplia, en conocer el derecho como ordenación social que se expresa a través de diferentes grados de institucionalización y se encuentra enraizado en las prácticas sociales, en medio de las cuales emerge como fenómeno social autónomo; una segunda, más circunscrita al problema de la constitución, que comprende el pluralismo como reconocimiento de una pluralidad de discursos constitucionales competentes o de autoridades competentes en un mismo territorio; $y$, además, una tercera, en un plano global, que resulta del esfuerzo por suministrar parámetros teóricos para lidiar con la pluralidad de mecanismos regulatorios emergentes, sea como desdoblamiento del derecho internacional, sea como espacios independientes de los mecanismos tradicionales de regulación interestatal.

La discusión en torno del pluralismo, desde su origen, se ve atravesada por dos cuestiones que se encuentran presentes en mayor o menor medida, y que pueden ser formuladas en los siguientes términos: (i) ¿cuáles son los criterios que pueden ser utilizados para diferenciar el derecho de las demás normas sociales en general?; y, (ii) ¿es posible (deseable) utilizar algún criterio para "ordenar" estas pluralidades normativas cuando entran en colisión? 
La primera cuestión, referida a la búsqueda de criterios para la identificación del derecho, se presenta cuando se pretende comprender el grado de exigibilidad de las expectativas contrafácticas y las interacciones existentes entre programas normativos distintos (moral, religión, derecho estatal, derecho no estatal, convención social). Esta controversia, que no se circunscribe únicamente a los fueros jurídicos, también dividió a sociólogos, antropólogos y politólogos desde el comienzo del auge de las investigaciones sobre el pluralismo jurídico. De esta forma, el debate es atravesado por dos perspectivas sociológicas opuestas: la comprensión del derecho como "patrón de conducta" y la comprensión del derecho como "coerción institucionalizada" (Tamanaha, 1995).

La segunda cuestión, relativa a la plausibilidad de la adopción de algún metacriterio para la solución de conflictos, será específicamente enfrentada más adelante (secc. 3). Mientras tanto, vale la pena llamar la atención sobre sus implicaciones con relación al problema de la soberanía (capacidad de decisión). Cuando se renuncia (aunque sea parcialmente) a un metacriterio basado en la autoridad territorial se produce, al mismo tiempo, tanto una especie de renuncia al monopolio estatal en cuanto a la afirmación coercitiva del derecho (ya que la normatividad que transciende los dominios del Estado "debe" tener su normatividad reconocida), como una especie de renuncia al monopolio de los tribunales nacionales (en especial de los tribunales constitucionales) en la resolución definitiva de los conflictos en torno del derecho aplicable.

Las diferentes perspectivas que toman seriamente el pluralismo jurídico pugnan por el reconocimiento de una auténtica normatividad jurídica más allá del derecho estatal. Tales perspectivas reúnen abordajes eminentemente descriptivos, al igual que otros con pretensiones normativas. Estas últimas pretenden inferir, de estas constelaciones de reglas de conducta con pretensiones de normatividad, elementos que permitan un enfrentamiento normativo de las colisiones potenciales y concretamente verificadas.

Con igual pretensión normativa, Klaus Günther (2008) reflexiona sobre los efectos de los desdoblamientos de esta realidad para la teoría del derecho. Con este propósito el autor identifica dos dificultades que exigen el desarrollo de nuevas categorías teóricas: la imposibilidad de construcción de un concepto uniforme sobre el derecho, ya que el sistema jurídico (antes lógica y jerárquicamente diferenciado) se convierte en una "pluralidad de regímenes jurídicos"; y la pérdida de fuerza persuasiva del ideal de plenitud del sistema (ideal este que reposa sobre la idea de representatividad/delegación y de identificación entre los autores y destinatarios de la norma) ${ }^{6}$.

$6 \quad$ En palabras del autor: "Political legislation by general and coherently ordered legal norms and a legal adjudication based on the coherent interpretation of legal norms and precedents which all belong to one legal system seems to be more and more replaced by fragmented 
De acuerdo con el autor, estos desafíos exigen el redimensionamiento estructural de la teoría general del derecho (y, por consiguiente, del propio constitucionalismo) a fin de que esta substituya su modelo de referencia fundado en el ideal de Estado territorial (constituido a partir de un único legislador legítimo y de un sistema de normas y precedentes), teniendo en la mira la incorporación de una postura de complementariedad de las perspectivas suscitadas por el pluralismo (Günther, 2008, 16).

Esto sería posible, prosigue el autor, mediante el reconocimiento de que, "a partir de un punto de vista interno ${ }^{7}$, los actores involucrados en las varias y multinivel redes de interlegalidad todavía se comunican teniendo por base, al menos hipotéticamente, un concepto uniforme de derecho". Concepto este que se traduce en un metalenguaje que contienen conceptos y reglas jurídicas básicas, como el "concepto de derechos y de procedimientos ecuánimes, y los conceptos de sanción y de incumbencia" (Günther, 2008, 16).

Sin pretender enfrentar en profundidad la cuestión, la premisa que se infiere a partir de esta presentación puede ser sintetizada en los siguientes términos: el derecho, como práctica discursiva (re)productora de expectativas en torno de la regulación social, se construye en múltiples fora y mediante grados de institucionalidad variados, razón por la cual resulta inaceptable su clausura (del derecho) bajo los auspicios del modelo de Estado-nación.

Esto resulta no solamente de las "exigencias" del discurso racional, sino, sobre todo, del reconocimiento de la multiplicidad de actores involucrados, de la diversidad de espacios productores de derecho (con lenguajes propios) y de las exigencias de legitimidad material que amplían vertiginosamente el auditorio concreto de los destinatarios de los discursos institucionales producidos acerca del derecho.

Es en este sentido que se asume, en este trabajo, que ante el ideal regulador de unidad sistémica del derecho centrado en el Estado (State-centredness), sin dispensar el atributo regulador de la noción de unidad, el constitucionalismo responde como una propuesta de unitas multiplex, o sea, de una unidad en la multiplicidad/diversidad. De esta forma, la constitución se asume como un punto de convergencia de una pluralidad de discursos sobre la propia constitución ${ }^{8}$.

areas of self-regulation, practised by norm giving actors who have empowered themselves and who enact norms with different degrees of generality and scope" (GüNTHER, 2008, 7).

7 Haciendo referencia a la distinción de Hart entre punto de vista interno y externo al sistema jurídico, el autor propone una especie de reconciliación (relación de complementaridad) entre el pluralismo jurídico y la teoría del derecho. Esto es posble cuando se percibe el potencial descriptivo suministrado por el pluralismo jurídico de las mútliples interacciones y negociaciones en torno de disposiciones jurídicas que postulan normatividad; y, a su turno, la necesidad de hacer operativos los diferentes procedimientos (estatales o no) para la toma de decisiones y para la salvaguardia, en especial, de terceros afectados por estos procesos decisorios.

8 De cierta forma, es este el sentido empleado por Gustavo Zagrebelsky cuando defiende el surgimiento de una concepción de soberanía centrada en la constitución, por medio de la cual, 
La propuesta de transición aquí delineada puede ser sintetizada en los siguientes términos.

TABLA 1

UNIDAD SISTÉMICA Y UNITAS MULTIPLEX

\begin{tabular}{|c|c|}
\hline UNIDAD SISTÉMICA (STATE-CENTREDNESS) & UNITAS MULTIPLEX (NO DEPENDIENTE DEL ESTADO) \\
\hline $\begin{array}{l}\text { - Derecho estatal (producido o reconocido } \\
\text { por el Estado). } \\
\text { - Teoría rígida de las fuentes del derecho. }\end{array}$ & $\begin{array}{l}\text { - Fuentes normativas autónomas y dotadas } \\
\text { de emergencia espontánea. } \\
\text { - Carácter dinámico de las fuentes. }\end{array}$ \\
\hline
\end{tabular}

Una vez fijados los trazos constitutivos de la noción de unitas multiplex, el paso siguiente consiste en enfrentar el desafío relativo a la falta de metacriterio(s) de ordenación resultante del colapso del ideal de ordenación jerárquica construido sobre bases territoriales (modelo de Estado territorial soberano).

\section{DEL PRINCIPIO DE JERARQUIZACIÓN NORMATIVA}

A LA IDEA-FUERZA DE HETERARQUÍA

Como ya se señalara, esta sección pretende delinear los contornos de un principio de organización que permita múltiples formas de interacción no estructuradas previamente. Este ideal de regulación debe permitir la estructuración de relaciones contingentes, dinámicas y asimétricas entre regímenes jurídicos dispares y entre discursos constitucionales en competencia. Se trata, pues, de la substitución de la pretensión de ordenación jerárquica por nuevas formas de estructuración "heterárquica".

Antes de avanzar en la sección es imprescindible precisar los contornos de la idea de heterarquía, teniendo en cuenta la multiplicidad de sentidos en que es empleada por la literatura. Ino Augsberg (2009) identifica tres diferentes usos: (i) empírico, (ii) sociólogo y (iii) epistemológico.

De acuerdo con el primer uso, el empírico, la expresión "heterárquica" es utilizada para describir formas de organización política no estructuradas jerárquicamente; su principal preocupación consiste en diferenciar esta "nueva" estructura de ordenación de aquellas tradicionalmente asociadas a los Estado-nación, o aun, resaltar la existencia de diversos parámetros y formas de interacción no jerárquicas entre los niveles de regulación interrelacionados.

la constitución se convertiría en un punto de llegada, en una pluralidad de valores constituidos hacia donde deben converger (y entrecruzarse) los diferentes discursos sobre la constitución. De acuerdo con el autor, "[p]ara darse cuenta de esta transformación ya no puede pensarse en la Constitución como centro del que todo derivaba por irradiación a través de la soberanía del Estado en que se apoyaba, sino como centro sobre el que todo debe converger; es decir, más bien como centro a alcanzar que como centro del que partir" (ZAGREBELSKY, 1995, 32). 
A continuación, en el uso sociológico la ordenación heterárquica representa la única forma posible de comprender las relaciones sociales en virtud del proceso acelerado de transformación de las sociedades contemporáneas. De esta manera, la forma de estructuración heterárquica se traduce en modelos teóricos a partir de los cuales son producidos elementos conceptuales que suministran instrumental adecuado a la comprensión tanto de los procesos de interrelación como de los procesos de colisión entre sistemas; estos últimos, articulados de manera dinámica y flexible.

Por último, de acuerdo con el tercer uso, el epistemológico, la comprensión de formas heterárquicas de relación resulta de la necesidad de un cambio más profundo en las condiciones de posibilidad de producción del propio conocimiento. En efecto, la necesidad de superación de la racionalidad moderna se impone como una respuesta a los desafíos suscitados por las diferentes paradojas contemporáneas. Así, tomando como base esta posibilidad, la idea de heterarquía resulta ser otra posibilidad epistemológica de comprensión de la forma de organización de lo político y del derecho?.

Ahora bien, con relación a la temática enfrentada en este trabajo, ¿cuáles son las implicaciones de la idea de heterarquía?

De lo dicho hasta aquí se puede inferir que la noción de heterarquía se traduce en la existencia de una diversidad de intereses, actores y recursos, sin que exista entre ellos el establecimiento, ex ante, de relaciones de relevancia o de preeminencia a favor de uno o de otro. Esto no significa que las relaciones establecidas entre los actores distintos son rigurosamente simétricas, sino solo que ninguno de ellos es capaz de alcanzar, por sí solo, sus objetivos; resulta de ahí la relación de recíproca dependencia entre los actores.

Sobre la base de la lección de Tamara Kovziridz (2009), la contraposición entre ordenación heterárquica y ordenación jerárquica, tomadas como tipos ideales, puede formularse en los siguientes términos.

9 Pueden ser identificadas tres perspectivas diferentes: a) de acuerdo con la teoría de sistemas, como una forma de ordenación resultante de procesos de des-jerarquización de la forma de comprender las organizaciones sociales; b) como la lógica que dirige la teoría de la estructuración social, desarrollada por Kyriakos Kontopoulos (1993, 209-267), para quien la lógica heterárquica permite la creación de una matriz conceptual apta no solo para describir los diferentes tipos de estructura y niveles estructurales, sino igualmente para suministrar parámetros para la conjunción de relaciones establecidas no solamente en el mismo nivel de ordenación sino, sobre todo, entre estructuras institucionales ubicadas en niveles diferentes; c) como indisociablemente ligada a la noción de sociedad en red formulada, entre otros, por Manuel Castells (2003), para quien la negación de relaciones estructuradas jerárquicamente representa el punto de partida para el desarrollo de relaciones sociales articuladas a partir de nociones como nudos y enlaces. Llevadas estas ideas al campo de la regulación, la idea de heterarquía aparece como la única forma de articular las relaciones que se procesan simultáneamente tanto en el ámbito de las comunidades políticas estatales como en otras comunidades no estructuradas a partir de fronteras y de identidades claramente definidas. 
TABLA 2

DISTINCIONES ENTRE LAS ORDENACIONES JERÁRQUICA Y HETERÁRQUICA

\begin{tabular}{|c|c|c|}
\hline & JERARQUÍA & HETERARQuía* \\
\hline Tipos de dependencia & Dependencia unilateral & Dependencia recíproca \\
\hline $\begin{array}{l}\text { Canales de } \\
\text { comunicación }\end{array}$ & $\begin{array}{l}\text { Comunicaciones (unilaterales } \\
\text { o bilaterales) estructuradas } \\
\text { verticalmente (top-down y bottom- } \\
\text { up) entre los niveles }\end{array}$ & $\begin{array}{l}\text { Coexistencia de comunicaciones } \\
\text { unilaterales, verticales y } \\
\text { multilaterales entre los niveles }\end{array}$ \\
\hline $\begin{array}{l}\text { Dirección de las } \\
\text { relaciones entre los } \\
\text { niveles }\end{array}$ & $\begin{array}{l}\text { Estructura piramidal. Relaciones } \\
\text { verticales }\end{array}$ & $\begin{array}{l}\text { Estructura no piramidal. } \\
\text { Relaciones tanto verticales } \\
\text { como horizontales }\end{array}$ \\
\hline Tipos de coordinación & De arriba hacia abajo & $\begin{array}{l}\text { Varios tipos de coordinación } \\
\text { posibles }\end{array}$ \\
\hline $\begin{array}{l}\text { Distribución de poderes } \\
\text { y atribuciones }\end{array}$ & $\begin{array}{l}\text { Altamente asimétrico. } \\
\text { Concentración de poderes en los } \\
\text { niveles superiores. }\end{array}$ & $\begin{array}{l}\text { Diferentes grados de simetría y } \\
\text { asimetría }\end{array}$ \\
\hline Grado de flexibilidad & $\begin{array}{l}\text { Una vez establecido, relativamente } \\
\text { estático y rígido }\end{array}$ & $\begin{array}{l}\text { Flexible y más fácilmente } \\
\text { adaptable en el tiempo }\end{array}$ \\
\hline $\begin{array}{l}\text { Tipos de estructura y de } \\
\text { ligación }\end{array}$ & $\begin{array}{l}\text { Estructurada en varios niveles, } \\
\text { pero los niveles inferiores se } \\
\text { encuentran englobados (nested) por } \\
\text { los superiores }\end{array}$ & $\begin{array}{l}\text { Estructurada a través de varias } \\
\text { entidades ligadas unas a otras (y } \\
\text { no abarcadas unas por otras) }\end{array}$ \\
\hline
\end{tabular}

"La autora utiliza la terminología "interdependencia". Para guardar congruencia con la propuesta de esta sub-sección y considerando la aproximación entre la definición de interdependencia y de jerarquía presentada por la autora, se optó por adaptar la expresión como "heterarquía".

Finalmente, considerando los discursos de resignificación del constitucionalismo, es importante referirse a la posibilidad de aplicación de la idea de heterarquía tanto a las relaciones de ordenaciones intraestatales como a las relaciones a escala global.

Con relación a los escenarios de regulación que transcienden las fronteras territoriales del Estado, considerando el escenario tradicional de las relaciones interestatales, las relaciones entre los actores estatales siempre fueron concebidas en términos no jerárquicos. En este contexto, la mera posibilidad de concebir estructuras de gobernanza solo puede ser postulada en caso de adopción de modelos no jerarquizados de interrelaciones ${ }^{10}$. Estas estructu-

10 En sentido similar, se reproduce aquí la descripción pertinente hecha por Volker Rittberger $(2008,16)$ : "The concept of heterarchy is meant to describe the existence of an increasingly dense network of institutions of global governance, created and maintained by public and private actors, and aimed at the rules-based collective management of transovereign problems through horizontal policy coordination and cooperation where different groups of actors (States, intergovernmental organizations, civil society organizations, transnational corporations/private sector actors) are sensitive to each other's values and interests and dependent on one another 
ras, por lo tanto, son concebidas como redes desprovistas de un centro de imputación de autoridad y son descritas por Jochen von Bernstorff (2004, 258) en los siguientes términos:

El concepto de red, en general, se vincula a las relaciones jerárquicas entre actores múltiples. La unidad de esas relaciones es entendida como red y, a su vez, las redes son caracterizadas por acoplamientos no jerárquicos y relativamente débiles entre sus elementos constitutivos (nudos y enlaces). Al mismo tiempo, una red, como un todo, depende de esos nudos. Por consiguiente, cada nudo se presenta como interdependiente con relación a los demás nudos de la red. Respecto de esta concepción no centralizada, la autoridad es entendida como compartida a lo largo de la red ${ }^{11}$.

De esta forma, comprender estas relaciones en términos heterárquicos implica reconocer, de un lado, un movimiento de expansión (cuantitativa y cualitativa) de la densidad normativa en estos espacios de regulación (transnacionales e interestatales), de otro lado, la necesidad de lidiar con la insurgencia de patrones normativos que tornan las redes y los espacios de actuación de los actores internacionales mucho más "viscosos" que otrora (Zacher, 2000).

La cuestión más sensible, y que interesa más de cerca a esta investigación, consiste en la discusión sobre la posibilidad de comprender la ordenación intraestatal en términos heterárquicos. Con respecto a este nivel de análisis, sin embargo, hay aun menos acuerdo en cuanto al significado y alcance de esta expresión.

En el espacio europeo, por cuenta de la densidad de las normas comunitarias y de la dinámica actuación de sus cortes regionales (Tribunal de Justicia Europea y Corte Europea de Derechos Humanos), al referirse a la organización heterárquica (o al pluralismo constitucional) se está reflexionando sobre una especie de construcción de un espacio de cooperación regional en el que el derecho europeo representa, por lo menos, un espacio de articulación entre el derecho internacional y los espacios de regulación nacionales. De esta forma, la disposición jurídica europea se impone (no sin contestaciones) desde fuera a las autoridades nacionales, las cuales, por consiguiente, ven limitada su capacidad de tomar decisiones.

to achieve collective goals. The peculiarities of a heterarchical world order consist, inter alia, in the horizontal generation and implementation of norms and rules - which is not bound to a vertical, top-down policy process".

11 Traducción libre de: "The network concept in general focuses on heterarchical relationships between multiple actors. The unity of these relationships is conceptualised as a network, and networks are characterised by a non-hierarchical and relatively loose coupling (nodes) of their constituent elements. At the same time, however, a network, as a whole, depends on these nodes. Thus, every node is interdependent of the other nodes of the network. From this a-centric conception, it follows that authority is conceptualised as being shared along the network". 
En este ámbito, innumerables incumbencias, hasta entonces indisociables de la idea de soberanía, son expresamente transferidas a las instituciones comunitarias; $y$, con ello, inexorablemente ocurre un debilitamiento de las instituciones nacionales. Sin embargo, estos mecanismos de "transferencia" de atribuciones no se caracterizan por juegos de suma cero (zero sum game), puesto que la actuación comunitaria depende del desenvolvimiento de numerosos (y estrechos) mecanismos de cooperación y de coordinación de las actividades domésticas por parte de las instituciones nacionales.

Dicho de otro modo, a pesar del proceso de integración supranacional, los Estados no son simplemente incorporados en una nueva estructura protofederativa. Al mismo tiempo que las instancias nacionales son forzadas a "considerar" elementos normativos que superan las fronteras territoriales nacionales, estas mismas instancias se imponen como importantes actores con alcance transnacional, ya que sus decisiones también impactan y "deben" ser consideradas en otros fora externos. Esta "nueva" dinámica pretende ser aprehendida, por ejemplo, por conceptos como el de "interconstitucionalidad" (Canotilho, 2008), el de "conexión causal" mediante "jurisprudencia constitucional cooperativa" (Zagrebelsky, 2008), el de "Estado constitucional cooperativo" (Häberle, 2007) o el de "transconstitucionalismo" (Neves, 2009).

No obstante, reconocer la ordenación constitucional como una estructura heterárquica implica ir más allá de la interrelación entre las instancias nacionales y comunitarias; implica, también, resignificar diversas dimensiones tradicionalmente confiadas a la autoridad constitucional.

El agotamiento de la idea de estructura jerarquizada para describir las relaciones intraestatales vienen siendo considerado en distintos análisis. Algunos de esos esfuerzos serán examinados rápidamente en lo que sigue.

(i) Análisis sobre asistencia recurrente en los sistemas constitucionales de situaciones en que no hay clara definición sobre el papel institucional que deben desempeñar los órganos constitucionales. De acuerdo con esta perspectiva, la coexistencia de competencia entre órganos constitucionales no se presenta como un problema en sí, al contrario, el eventual choque entre autoridades igualmente legítimas a veces posibilita importantes redimensionamientos institucionales en momentos de crisis institucional.

(ii) Análisis sobre la imposibilidad de comprender las relaciones federativas en términos estrictamente jerárquicos considerando tanto la existencia de espacios de autonomía constitucionalmente conferidos a las unidades territoriales, como las necesidades concretas de acomodación de tensiones a partir de diferentes mecanismos de presión de abajo hacia arriba (bottom up).

(iii) Análisis sobre la artificialidad de la idea de jerarquía constitucional, considerando el carácter siempre creativo y transformador de las concretizaciones constitucionales y la apertura constitucional a las fuerzas actualizadoras de la esfera pública; es en este contexto que se postula la idea de que la cons- 
titución resulta del entrecruzamiento de innumerables discursos pertinentes sobre la constitución con pretensión de afirmarse frente a los demás.

Cada uno de los elementos antes expuestos representa una importante dimensión que desafía la idea de jerarquización modernamente asociada al Estado y a los ordenamientos jurídicos unificados por la constitución nacional. Además, cuando se reconoce aquí que la ordenación doméstica debe ser comprendida como una ordenación heterárquica, se defiende la necesidad de un cambio más radical en la forma de ordenación diseñada por la constitución ${ }^{12}$.

La transición hasta aquí presentada puede ser compendiada en el siguiente cuadro.

\begin{tabular}{|c|c|}
\hline ORDENACIÓN JERÁRQUICA & ACOMODACIÓN “HETERÁRQUICA” \\
\hline $\begin{array}{l}\text { - Punto de imputación de validez } \\
\text { - ¿Quién decide? Intérprete último }\end{array}$ & $\begin{array}{l}\text { - Diferentes niveles jurídicos multinivel y fraccionados } \\
\text { - Autoridad compartida }\end{array}$ \\
\hline
\end{tabular}

Así, es posible establecer una comparación entre estos órdenes en los siguientes términos: la ordenación jerárquica presupone la existencia de un único punto de imputación de validez, al contrario de la ordenación heterárquica que se sostiene en diferentes regímenes jurídicos multinivel. Sus conflictos, por tanto (entre normas y entre autoridades decisorias), dependen, muchas veces, de la cooperación y del reconocimiento mutuo; y el problema acerca del intérprete último (soberanía) termina siendo substituido, en cierto sentido, por una idea de divisiones de autoridad.

Por lo tanto, el hecho de compartimiento de autoridad presupone el reconocimiento de la imposibilidad de definir previamente quién es la autoridad competente para decidir, en última instancia y de manera inapelable, sobre las tensiones que involucran estos múltiples regímenes.

\section{DE LA COHERENCIA INTRASISTEMÁTICA A LA EXIGENCIA DE COMPATIBILIZACIÓN: EN BUSCA DE NUEVOS PARÁMETROS PARA LA SUPRAORDENACIÓN EN ESTRUCTURAS HETERÁRQUICAS}

Cuando se dice que es imposible entender estas múltiples relaciones entre los sistemas normativos desde un metacriterio de ordenación basada en la noción de jerarquía surge una dificultad esencial: ¿cómo lidiar con los conflictos (entre autoridades y normas) que surgen en estos espacios de interacción?

12 Esto implica: "en la emergencia de otra orden que opera mediante colisiones de normas en medio a un derecho plural en proceso de sedimentación; y que altera el papel del Estado de derecho (intra)estatal de forma de conjugar su predisposición para la observación de los intereses extra-estatales con la posibilidad de expansión de la validez y efecacia de sus ordenaciones más allá de sus propis fronteras" (LADEUR, 2010,23). 
Debido a la crisis de la noción ordenadora de territorio, los ideales de unidad, de plenitud y de coherencia intrasistemática de los ordenamientos jurídicos ceden el lugar a nuevos desafíos de compatibilización entre los órdenes jurídicos en colisión. Las antinomias y la coexistencia de autoridades superpuestas se presentan como una realidad constitutiva del sistema, por lo tanto, ya no pueden ser entendidas como problemas que deben superarse. En otras palabras, el derecho no es más capaz de proveer reglas claras para la solución de los conflictos normativos intersistémicos, ya sea en cuanto a la regla aplicable, ya sea en cuanto a la autoridad competente para poner fin a la controversia.

Las diferentes narrativas sobre los procesos de globalización y la expansión de los espacios regulatorios ubicados fuera del Estado llevan a dos constataciones desconcertantes: de una parte, los órdenes jurídicos se encuentran inexorablemente entrelazados, de otra, la constitución no es más capaz de operar como fuente de demarcación de los límites jurídicos del derecho nacional.

Teniendo en cuenta el carácter normativo de los discursos constitucionales y la característica del constitucionalismo de reinventarse a sí mismo frente a los nuevos problemas, múltiples son los esfuerzos teóricos que pretenden identificar parámetros normativos para hacer frente a este choque de los sistemas mediante la estipulación de metacriterios de autoridad.

Estos intentos teóricos de establecer criterios para ordenar las relaciones existentes entre los órdenes jurídicos interno y externo (tanto interestatal como transnacional) pueden ser agrupados en dos perspectivas contrapuestas: constitucionalista y pluralista (Krisch, 2009). Dos aspectos deben ser tenidos en cuenta respecto de esta oposición: el reconocimiento (o no) de la existencia de un elemento común de conexión entre los sistemas (common framework) y la existencia (o no) de algún principio de ordenación en la forma como se da la articulación entre los sistemas (ordenación jerárquica o heterárquica).

De acuerdo con Nico Kirsch $(2009,2)$, mientras los constitucionalistas generalmente defienden la existencia de principios sustantivos compartidos entre los distintos ámbitos de ordenación y su articulación a través de relaciones de supraordenación top-down (reconocimiento de alguna forma de constitucionalización del derecho internacional), los pluralistas "prefieren ver el mundo post-nacional como caracterizado por la heterarquía, por una interacción de diferentes subórdenes que no están sujetos al mismo patrón de reglas jurídicas, sino dotados de formas más abiertas y políticas"13.

A su vez, en relación con el último grupo (perspectivas pluralistas), a pesar de compartir entre sí una actitud de negación de cualquier principio

13 Traducción libre de: "prefer to see the postnational realm as characterised by heterarchy, by an interaction of different sub-orders that is not subject to common legal rules but takes a more open, political form". 
de organización jerárquicamente estructurado, no está de acuerdo en cuanto a la existencia de algún cuadro común de referencia (commom framework), pudiendo ser divididos sus integrantes en institucionalistas y sistémicos.

Los primeros (pluralistas institucionalistas) tienden a admitir la existencia de algún mecanismo institucional de articulación entre los regímenes diferenciados, o aun, la posibilidad de articulación sustantiva de los discursos entre los sectores involucrados (Kirsch, 2009, 17). De esta forma, esta variación institucionalista del pluralismo se presenta mucho más como una continuación de la propuesta constitucionalista que, propiamente, como una alternativa a aquel modelo (Kirsch, 2009, 18).

Por otro lado, los segundos (pluralistas sistémicos ${ }^{14}$ ) se muestran escépticos respecto a cualquier pretensión de ordenación que se asemeje al trasplante al ámbito de regulación global de resquicios de modelos organizativos centrados en el Estado (Kirsch, 2009, 17). Estos teóricos se co-asocian en torno a una especie de gerencia descentralizada de la diversidad, razón por la cual, a pesar de las incertezas suscitadas en virtud de las colisiones cada vez más frecuentes, presentan ventajas innegables que no pueden ser, de plano, despreciadas. De acuerdo con sus características señaladas por Nico Krisch (2009, 19-28), estas perspectivas confieren mayor capacidad de adaptación, permiten más espacio para contestación y, además, suministran mecanismos dinámicos de check and balances.

Al confrontarse las diferentes perspectivas teóricas, lo que se percibe es que la diferencia que las contrapone es mucho más cuantitativa que cualitativa. Dicho de otro modo, es posible articular estos modelos en una especie de combinación que gravita en torno de dos extremos configurados por las versiones fuertes de la perspectiva constitucionalista y del pluralismo sistémico, como se puede observar en la figura siguiente.

FIGURA 1

ESCALA DE TRANSICIÓN ENTRE PERSPECTIVAS CONSTITUCIONALISTA Y PLURALISTA

\begin{tabular}{|ccc|}
\hline $\begin{array}{c}\text { Constitucionalismo } \\
\text { ordenación jerárquica) }\end{array}$ & $\begin{array}{c}\text { Pluralismo constitucional } \\
\text { (cuadro común institucional } \\
\text { o procedimental + no } \\
\text { jerarquía) }\end{array}$ & $\begin{array}{c}\text { Plausencia de cualquier cuadro } \\
\text { común + no jerarquía) }\end{array}$ \\
\hline
\end{tabular}

Aunque sea bastante ilustrativa la realización de un cotejo analítico entre algunas de estas propuestas teóricas (en especial, con relación a la forma en

14 La expresión fue acuñada por Neil MacCormick (1993). 
que ellas enfrentan las cuestiones del cuadro común y de la ordenación), este esfuerzo excede los límites de este trabajo.

La perspectiva adoptada acá se ubica en el ámbito del llamado "pluralismo institucional", en especial, defiende la necesidad de identificación de las bases de un compromiso existencial con la promoción de los "espacios de hibridación"15 (Bermann, 2002; 2007) y de la posibilidad de múltiples enlaces comunitarios expresados a través de la máxima postulada por Michel Rosenfeld (2008) en torno de un "pluralismo comprensivo".

El principal motor que impulsa esta perspectiva consiste en la existencia de un compromiso existencial en preservar (y promover) las comunidades y compromisos normativos generados a través de múltiples interacciones (formación dinámica de selve y others) (Rosenfeld, 2010). Por lo tanto, se confiere un énfasis excesivo a la dimensión institucional en que las interacciones normativas son realizadas. Sin embargo, la cuestión central no consiste solamente en la identificación de procedimientos y mecanismos de apertura y diálogo, se busca discutir en qué medida es posible formular una concepción de jurisdicción que, de alguna forma, sea dislocada de la noción de territorio (Berman, 2002; 2005), construyéndose en términos "pluralistas y cosmopolitas" 16 .

Con relación a la propuesta de Paul S. Berman (2007), en la que, a pesar de su esfuerzo en definir un modelo analítico a partir del cual considera posible lidiar con esta nueva gama de conflictos normativos y el compromiso con el carácter híbrido de los espacios de regulación, aún no es posible estructurar estas interacciones a partir de una matriz teórica capaz de lidiar, concomitantemente, con diseños institucionales y con prácticas discursivas tan dispares como las que marcan la actuación de los diferentes actores y escenarios de interacción normativa (Berman, 2007, 1159).

$\mathrm{Si}$, por un lado, este artículo rechaza la alternativa kelseniana de un monismo internacionalista que pasaría a concebir la relación entre orden internacional e interno a partir de una relación de supraordenación, por otro lado, no es capaz de identificar, con precisión, los contornos de este nuevo modelo teórico de interacción (¿tal vez porque es imposible comprender estos fenómenos a partir de un único modelo teórico de interacción?).

15 De acuerdo con el autor, estos espacios híbridos ocurren "where more than one legal, or quasi-legal, regime occupies the same social field" (BERMAN, 2007, 1158), con una variedad de formas que "overlapping legal systems interact with each other and observed that the very existence of multiple systems can at times create openings for contestation, resistance, and creative adaptation" (BERMAN, 2007, 1159).

16 En palabras del autor: "cosmopolitan because it recognized the possibility that people can hold multiple, sometimes nonterritorial, community affiliations; and pluralist because it acknowledged that forms of legal (or quasi-legal) jurisdiction can be asserted by communities beyond those represented by official statesanctioned courts" (BERMAN, 2005, 1821). 
Por lo tanto, la máxima aquí postulada de "exigencia de compatibilización normativa" como directriz que pretende suplantar el dogma de la "coherencia intrasistemática", solo puede ser formulada bajo las bases de un compromiso de convivencia y de preservación de las diferencias. A lo sumo, es posible identificar esta postura que se materializa en diferentes prácticas discursivas y que se instrumentaliza a través de mecanismos desarrollados de disminución de las tensiones y conflictos. Se presenta, por lo tanto, tan solo como una directriz que apunta a posibles caminos y alternativas. Nada más.

\section{A MODO DE CONCLUSIÓN: PLURALISMO CONSTITUCIONAL COMO RESPUESTA POSIBLE A LAS PRESIONES SOBRE LA CONSTITUCIÓN NACIONAL}

El escenario de transición antes descrito (del monismo al pluralismo, de la ordenación jerárquica a la heterárquica, y del debate en torno de la existencia de metacriterios para la solución de conflictos) resulta en aquello que aquí es llamado pluralismo constitucional. En otras palabras, resulta de la multiplicación de los discursos sobre la constitución producidos en diferentes centros decisorios situados en espacios que trascienden los límites geográficos del Estado y que se presentan como narrativas resultantes de los discursos institucionales de las instancias decisorias estatales.

Esta multiplicación de discursos, como se dijo, resulta del surgimiento de espacios decisorios más allá del Estado ${ }^{17}$, es impulsada por la actuación de numerosos agentes (estatales y no estatales) y revela la imposibilidad de definición previa tanto de la instancia decisoria competente como de la regla de derecho aplicable a la situación concreta. En este contexto, el conflicto, la composición política y los discursos de legitimación material se revelan como características inexorables de este nuevo escenario de regulación constitucional y le confieren particular acento al papel desempeñado, en especial, por los diferentes tribunales.

Así, los diferentes órganos decisorios (resolución y arbitramento de conflictos) acaban ocupando posiciones centrales en el proceso de delimitación de las fronteras del derecho. Esto es así porque les incumbe a los tribunales (sobre todo aquellos cuya decisión se presenta formalmente inapelable ${ }^{18}$ ), de

17 Los espacios supranacionales, intergubernamentales e interestatales se constituyen mediante intrincados procesos de negociación y de deliberación política que todavía tienen en los agentes políticos de los Estados sus principales actores. Los espacios de regulación privados no-estatales, a su vez, se institucionalizam y construyen sus cadenas normativas y decisorias independientemente del Estado y, en muchas situaciones, con pretensiones concurrentes con relación a las instancias de regulación oficiales.

18 Los órganos decisorios internacionales y supranacionales no realizan un auténtico trabajo de revisión judicial de las decisiones proferidas en otras instancias normativas. Esto es así porque, técnicamente, no existe relación de subordinación jerárquica entre instancias decisorias 
cara a los conflictos concretos suscitados ante ellos, tanto la identificación del derecho aplicable (law finding) como la definición del propio ámbito de competencia (Kompetenz-kompetenz).

Estas características, entre otras, les permiten a los tribunales institucionalizar la porosidad entre los sistemas y conferir una capacidad de adaptabilidad del respectivo sistema jurídico al entorno que las instituciones tradicionales (teniendo como referencia el derecho estatal y el derecho internacional) no son operativamente capaces de suministrar.

Paralelamente al proceso de "trueque de soberanía"19 (operado a nivel político) ocurren, en el ámbito de los tribunales, movimientos similares de compresión y de expansión de su autoridad. Estos procesos se materializan mediante el reconocimiento de una pluralidad de discursos constitucionales.

La multiplicación de discursos sobre la constitución genera, sin embargo, paradojas, contradicciones y ambigüedades en dos niveles distintos: (1) con respecto a la propia idea de constitución y (2) con respecto a la autoridad, en este caso, del poder judicial nacional (sobre todo, de los tribunales constitucionales en la condición de guardianes de la constitución).

(1) En cuanto a la fuerza sugestiva de la idea de constitución, el reconocimiento de una pluralidad de discursos constitucionales concurre para su propio fortalecimiento (más constitución), tanto en los espacios de regulación interna (desdoblamiento lógico de la supremacía constitucional) como en los espacios de regulación más allá del Estado (dislocamiento de la gramática de la constitución a otros ámbitos).

Este fortalecimiento, no obstante, genera dos problemas. De un lado, propicia un aumento exponencial de expectativas en torno de las promesas constitucionales, lo que a su vez puede llevar a la sobrecarga de expectativas y a la disfuncionalidad en relación con la constitución (menos constitución $)^{20}$. De otro lado, aumenta sobremanera los conflictos relacionados con las demandas por reconocimiento y por inclusión discursiva y los conflictos sobre

distintas. La estrategia para lidiar con la discusión sobre la existencia (o no) de inobservancia de las obligaciones internacionales asumidas y reguladas en el ámbito del régimen internacional en el cual se encuentra aquel órgano decisorio es tratar las decisiones producidas en otras esferas como "materia de hecho" (AHDieH, 2004).

19 Los dislocamentos de la capacidad regulatoria y de la atribución de la resolución de conflictos a instancias situadas fuera del Estado no autorizan que se hable de asalto a la soberanía, o de procesos de sujeción de la autoridad constitucional a elementos externos. Al contrario, tales procesos de transferencia de competencias por parte del Estado no se caracterizan por la eliminación de la importancia por él revestida; sino que se procesan por intermedio de intrincados procesos de negociación (CAPORASO, 2000) y trueques (LitFIn, 1997), mediante los cuales las interacciones y cambies entre los sistemas no ocurren ni unidireccional, ni jerárquicamente.

20 A este proceso de sobrecarga de expectativas en torno a la constitución y los riesgos asociados de disfuncionalidad operativa en sociedades periféricas, Marcelos Neves $(1994 ; 1995)$ lo denomina "constitucionalización simbólica". 
la naturaleza y el contenido de los discursos constitucionales vehiculados (muchas constituciones).

(2) En cuanto a la autoridad, el reconocimiento de múltiples centros decisorios (productores de discursos constitucionales) acaba produciendo instancias concurrentes y, en muchos casos, hace nacer, al menos, un compromiso de diálogo con estas instancias. Esto implica, en cierto sentido, una reducción del margen de autonomía de los tribunales y, por consiguiente, menos autoridad.

Por otro lado, la proyección de estas instancias de gramática constitucional ha autorizado que los propios tribunales nacionales ejerzan un control de legitimidad de las decisiones proferidas en otros ámbitos, siguiendo el ejemplo de las multicitadas decisiones de la Corte alemana (Solange I y Solange $\left.I I^{21}\right)$. De esta forma, al afirmar el control de legitimidad de las decisiones proferidas "del lado de fuera" (outside), los tribunales, por vías transversales, afirman su propia autoridad frente a estas instancias. Este hecho de compartir legitimidad genera, por consiguiente, más autoridad. Esta ambigüedad de fortalecimiento/debilitamiento de los tribunales mediante la multiplicación de los discursos constitucionales se expresa, ejemplarmente, por la propuesta de Armin von Bogdandy de acoplamiento o "sistemas de vinculaciones".

Lo que se pretendió defender en este trabajo es que, pese a la profundidad de la crisis experimentada, es posible sin embargo concebir la constitución como instrumento útil y apto para producir regulación en las comunidades políticas nacionales. Sus funciones clásicas pueden continuar siendo operativas, siempre que las mismas sean redimensionadas a partir de un compromiso existencial con el pluralismo. De esta forma, tanto las funciones de integridad como de integración pasan a ser redimensionadas a partir del reconocimiento de que la capacidad de producción de discursos constitucionales depende de su aptitud para producir (nuevos) puntos de convergencia material.

21 En ambos casos - Solange I (BVerfGE 37, 271 ss., de 1975) y Solange II (BVerfGE 75, 223 ss., de 1987), el Tribunal Constitucional Federal alemán reconoció ser competente para declarar la compatibilidad (o no) de la ejecución de actos concernientes al derecho comunitario secundario en clave de la Constitución alemana. El rasgo distintivo de estas decisiones corresponde a la postura adoptada por la Corte. En un primer momento, el Tribunal resaltó que en cuanto el derecho comunitario fuera caracterizado por un proceso de integración económica (y, por tanto, sin que el sistema se ocupara de tutelar los derechos considerados como fundamentales por el derecho constitucional alemán), la Corte siempre podría evaluar la compatibilidad del derecho comunitario en clave de las cláusulas de garantía provistas en el constitucionalismo alemán (clara postura de confrontación). Esta decisión tuvo una gran repercusión que acabó generando la retractación de las actuaciones de la Corte Europea. En la decisión siguiente, la línea del Tribunal alemán cambió. A pesar de continuar reconociendo la posibilidad de ejercer ese control, lo asume como residual, después de acoger la línea jurisprudencial asumida por la Corte Europea en el sentido de incorporar una gramática proteccionista de derechos fundamentales en el ámbito comunitario. De esta forma, el Tribunal alemán reconoce una especie de presunción en favor del derecho comunitario (clara posición de cooperación). 


\section{REFERENCIAS}

AhDien, R. B. (2004). Between dialogue and decree: International review of national courts. New York University Law Review. Vol. 79, n. ${ }^{\circ}$ 6, 101-228.

Augsberg, I. (2009). The relevance of network models within the juridic discourse. Empirical, sociological, and epistemological perspectives. German Law Journal. Vol. 10, n. ${ }^{\circ} 4,383-394$.

AvbeLu, M. (2006). The EU and the many faces of legal pluralism toward a coherent or uniform EU legal order? Croatian Yearbook of European Law and Policy, University of Zagreb. Vol. 2, 377-391.

Bastos Jr., L. M. P. (2000). Possui a União Eu ropéia uma autêntica constituição? Um breve esboço sobre a natureza jurídica dos tratados de integração e o problema da legitimidade democrática. Revista de Informação Legislativa, Brasília. Vol. 37, n. .o 147, 145-160.

Bastos Jr., L. M. P. (2011). Constituição, quo vadis? Por um constitucionalismo da diferença como resposta aos desafios contemporâneos à teoria constitucional. Tese em Direito. Programa de Pós-Graduação em Direito, Universidade Federal de Santa Catarina, Florianópolis.

Berman, P. S. (2002). The globalization of jurisdiction. University of Pennsylvania Law Review. Vol. 151, n. ${ }^{\circ}$ 2, 311-529.

Berman, P. S. (2005). Towards a cosmopolitan vision of conflict of laws: Redefining governmental interests in a global era. University of Pennsylvania Law Review. Vol. 153, n. $^{\circ} 6,1819-1882$.

Berman, P. S. (2007). Global legal pluralism. Southern California Law Review. Vol. 80, n. ${ }^{\circ} 6,1155-1237$.

Calliess, CH. (2009). Europe as Transnational Law - The Transnationalization of Values by European Law. German Law Journal. Vol. 10, n. ${ }^{\circ} 10$.

CAporaso, J. (2000). Changes in the Westphalian order: territory, public authority, and sovereignity. En: CAPORASO, J. (org.). Continuity and change in the Westphalian order. Malden, usA; Oxford, UK: Blackwell, 1-28.

Castells, M. (2003). A sociedade em rede. 7. a ed. São Paulo: Paz e Terra.

CоттіеR, Тн. (2009). Multilayered governance, pluralism, and moral conflict. Indiana Journal of Global Legal Studies. Vol. 16, n. ${ }^{\circ} 2,647-679$.

De Sousa Santos, B. (2000). Para um novo senso comum: a ciência, o direito e a política na transição paradigmática. São Paulo: Cortez. Vol. 1: "A crítica da razão indolente: contra o desperdício da experiência".

Dupret, B. (2007). Legal pluralism, plurality of laws, and legal practices: Theories, critiques, and praxiological re-specification. European Journal of Legal Studies, European University Institute. Vol. 1, n. ${ }^{\circ}$ 2. Disponible en: http://www.ejls.eu/1/14UK.pdf 
Fraser, N. (2009). Reenquadrando a justiça em um mundo globalizado. Lua Nova: revista de cultura e política. CEDEC, . $^{\circ} 77,11-39$.

Gomes Canotilho, J. J. (2008). "Brancosos" e interconstitucionalidade: itinerários dos discursos sobre a historicidade constitucional. 2. ${ }^{\text {a }}$ ed. Coimbra: Almedina.

Griffiths, J. (1986). What is legal pluralism? The Journal of Legal Pluralism and Unofficial Law, University of Birmingham. Vol. 24, 1-55.

Gunther, K. (2008). Legal pluralism or uniform concept of law? Globalisation as a problem of legal theory. No Foundations: Journal of Extreme Legal Positivism, Helsinki, n. ${ }^{\circ} 5,5-21$.

HäBERLE, P. (1998). Libertad, igualdad, fraternidad: 1789 como historia, actualidad y futuro del Estado constitucional. Madrid: Trotta.

HäBerle, P. (2007). Estado constitucional cooperativo. Rio de Janeiro: Renovar.

Joerges, Ch. (2005). Rethinking European Law's Supremacy: A Plea for a Supranational Conflict of Laws. EuI Working Paper Law, European University Institute, Florence, Paper n. ${ }^{\circ}$ 2005/12.

Joerges, Ch. (2006). Constitutionalism in Postnational constellations: Contrasting social regulation in the EU and in the wto. En: Joerges, C. y Petersmann, E-U. (eds.). Constitutionalism, Multilevel Trade Governance and Social Regulation. Oxford, Portland: Hart, 491-527.

Petersmann, E.-U. (2007). Conflict of laws as constitutional form: Reflections on the International Trade Law and the Biotech Panel Report. RECON Online Working Paper Series, Working Paper n. ${ }^{\circ}$ 2007/03. Disponible en: http://www.reconproject.eu/main. php/RECON _wp_0703.pdf?fileitem=5456959

Petersmann, E.-U. (2010). The idea of three-dimensional conflicts law as constitutional form. RECON Online Working Paper Series, Working Paper n. ${ }^{\circ}$ 2010/05, 2010. Disponible en: www.reconproject.eu/main.php/projects/portalprojec

KomÁrek, J. (2010). Institutional Dimension of Constitutional Pluralism. Eric Stein Working Paper, Czech Society for European and Comparative Law, Paper n. ${ }^{\circ}$ 3. Disponible en: http://www.ericsteinpapers.eu

Kontopoulos, K. M. (1993). The logics of social structure. Cambridge: Cambridge.

Koskenniemi, M. (2006). Fragmentation of internacional Law: difficulties arising from the diversification and expansion of International Law. Report of the Study Group of the International Law Commission. Geneva, 1 May-9 June and 3 July-11 August. Identificação: A/CN.4/L.682

KovzIridz, T. (2009). Hierarchy and Interdependence in Multi-level Structures: Foreign and European Relations of Belgian, German and Austrian Federated Entities. Brussels: vuB Press.

KRISCH, N. (2009). The case for pluralism in postnational law. LSE Law, Society and Economy Working Papers, London School of Economics and Political Science, n. ${ }^{\circ} 12$. Disponible en: ssrn.com/abstract $=1418707$ 
Kumm, M. (1999). Who is the final arbiter of constitutionality in Europe?: Three conceptions of the relationship between the German constitutional court and the European Court of Justice. Common Market Law Review. Vol. 36, n. ${ }^{\circ}$ 2, 351-386.

Kumm, M. (2006). Democratic constitutionalism encounters international law: terms of engagement. En: Choudhry, S. The migration of constitutional ideas. Cambridge: Cambridge, 256-293.

Kumm, M. (2009). The Cosmopolitan Turn in Constitutionalism: On the Relationship between Constitutionalism in and beyond the State. En: Dunoff, J. y Trachtman, J. (eds). Ruling the world? Constitutionalism, International Law, and Global Governance. Cambridge: Cambridge, 258-325.

Lachmayer, K. (2007). The international Constitutional Law approach: An introduction to a new perspective on constitutional challenges in a globalizing world. The Vienna Online Journal on International Constitutional Law. Vol. 1, n. ${ }^{\circ}$ 2, 91-99. Disponible en: http://www.icl-journal.com

Litfin, K. T. (1997). Sovereignty in world ecopolitics. Mershon International Studies Review, The International Studies Association. Vol. 41, n. ${ }^{\circ}$ 2, 167-204.

MacCormick, N. (1993). Beyond the Sovereign State. The Modern Law Review. Vol. 56, n. ${ }^{\circ} 1,1-18$.

MacCormick, N. (1995). The Maastricht-Urteil: Sovereignty now. European Law Journal. Vol. 1, n. ${ }^{\circ} 3,259-266$.

MacCormick, N. (1999). Questioning sovereignty: Law, State, and practical reason. Oxford: Oxford University Press.

Maduro, M. P. (2003). Contrapunctual law: Europe's constitutional pluralism in action. En: WALKer, N. (ed.). Sovereignty in transition. Oxford, Portland: Hart, 501-537.

Maduro, M. P. (2006). A constituição plural: constitucionalismo e União Européia. Cascais: Principia.

Maduro, M. P. (2008). Interpreting european law - Judicial adjudication in a context of constitutional pluralism. Working Paper IE Law School, European University Institute, WPLS08-02. Disponible en: http://ssrn.com/abstract=1134503

Maduro, M. P. (2009). Courts and Pluralism: Essay on a Theory of Judicial Adjudication in the Context of Legal and Constitutional Pluralism. En: Dunoff, J. y Trachtman, J. (eds.). Ruling the world? Constitutionalism, International Law, and Global Governance. Cambridge: Cambridge, 356-379.

Mayer, F. (2006). The European Constitution and the Courts. En: Von Bogdandy, A. y Bast, J. (eds.). Principles of European Constitutional Law. Max Planck Institute for Comparative Public Law and International Law. Oxford; Portland: Hart Publishing, 281-333.

Neves, M. (1994). A constitucionalização simbólica. São Paulo: Acadêmica.

Neves, M. (1995). Constitucionalização simbólica e desconstitucionalização fática: mudança simbólica da constituição e permanência das estruturas reais de poder. Revista Trimestral de Direito Público, São Paulo, n. ${ }^{\circ}$ 12, 156-167. 
Neves, M. (2009). Transconstitucionalismo. São Paulo: Martins Fontes.

PAuwelyn, Joost (2004). Bridging fragmentation and unity: International Law as a universe of inter-connected islands. Michigan Journal of International Law. Vol. 25, n. ${ }^{\circ}$ 4, 903-916.

Peters, A. (2006). Compensatory constitutionalism: The function and potential of fundamental international norms and structures. Leiden Journal of International Law, 19, n. $^{\circ} 3,579-610$.

Peters, A. (2007). The globalization of State constitutions. En: Nollkaemper, A. y NiJMA, J. (eds.). New perspectives on the divide between national and international law. Oxford: Oxford, 251-308.

Peters, A. (2009). The Merits of Global Constitutionalism. Indiana Journal of Global Legal Studies. Vol. 16, n. ${ }^{\circ}$ 2, 397-411.

Rittberger, V. (2008). Global Governance: From 'exclusive' executive multilateralism to inclusive, multipartite institutions. Tübinger Arbeitspapiere zur Internationalen Politik und Friedensforschung, Institut für Politikwissenschaft Eberhard-Karls-Universität Tübingen, Paper n. ${ }^{\circ}$ 52. Disponible en: http://www.uni-tuebingen.de/uni/spi/taps/tap52.pdf

Rosenfeld, M. (2010). The identity of the constitutional subject: Selfhood, citizenship, culture, and community. London-New York: Routledge.

SweEt, A. S. (2000). Governing with judges: constitutional politics in Europe. Oxford: Oxford.

Sweet, A. S. y Mathews, J. (2008). Proportionality, balancing and global constitutionalism. Columbia Journal of Transnational Law. Vol. 47, n. ${ }^{\circ}$ 1, 73-165.

Tamanaha, B. (1995). An analytical map of social scientific approaches to the concept of law. Oxford Journal of Legal Studies. Vol. 15, n. . 4, 501-535.

TAmanaha, B. (2008). Understanding legal pluralism: past to present, local to global. Legal Studies Research Paper Series, St. John's University School of Law, Paper n. ${ }^{\circ}$ 07-0080. Disponible en: http://ssrn.com/abstract=1010105

Van Aaken, A. (2009). Defragmentation of Public International Law Through Interpretation: A Methodological Proposal. Indiana Journal of Global Legal Studies. Vol. 16, n. ${ }^{\circ} 2,483-512$.

Von Bernstorff, J. (2004). The Structural Limitations of Network Governance: ICAnN as a Case in Point. En: Joerges, C.; Sand, I.-J. y Teubner, G. (eds.). Transnational Governance and constitutionalism. Oxford, Portland: Hart, 257-282.

Von Bogdandy, A. (2008). Pluralism, direct effect, and the ultimate say: On the relationship between international and domestic constitutional law. International Journal of Constitutional Law. Vol. 6, n. ${ }^{\circ}$ 3-4, 397-413.

Von Bogdandy, A.; Wolfrum, R.; Von Bernstorff, J.; Dann, P. y Goldmann, M. (eds.). (2009). The Exercise of Public Authority by International Institutions: Advancing International Institutional Law. Heildeberg: Springer. 
WALker, N. (2008a). Beyond boundaries disputes and basic grids: Mapping the global disorder of normative orders. International Journal of Constitutional Law. Vol. 6, n. ${ }^{\circ} 3-4,373-396$.

Walker, N. (2008b). Taking constitutionalism beyond the State. Political Studies. Vol. 56, n. $^{\circ} 3,519-543$.

Walker, N. (2009). Reframing the EU Constitutionalism. En: Dunoff, J. y Trachtman, J. (eds.). Ruling the world? Constitutionalism, International Law, and Global Governance. Cambridge: Cambridge, 149-176.

Wolkmer, A. C. (1997). Pluralismo jurídico: fundamentos de uma nova cultura no direito. São Paulo: Alfa e Ômega.

Zacher, M. W. (2000). Os pilares em ruína do templo de Vestfália. En: Rosenau, J.; Czempiel, E-O. (orgs.). Governança sem governo: ordem e transformação na política mundial. Brasília: UnB; Imprensa Oficial, 83-141.

Zagrebelsky, G. (1995). El derecho dúctil: ley, derechos y justicia. Madrid: Trotta.

Zagrebelsky, G. (2008). El juez en el siglo Xxi. Revista Iberoamericana de Derecho Procesal Constitucional, n. ${ }^{\circ}$ 10, 249-268. Disponible en: http://www.iidpc.org/revistas/10/ pdf/265_283.pdf 